\section{The influence of training-induced sarcomerogenesis on the history dependence of force}

\author{
Jackey Chen, Parastoo Mashouri, Stephanie Fontyn, Mikella Valvano, Shakeap Elliott- \\ Mohamed, Alex M. Noonan, Stephen H. M. Brown \& Geoffrey A. Power
}

Department of Human Health and Nutritional Sciences, College of Biological Sciences, University of Guelph, Guelph, Ontario, Canada

\section{Original Research Article}

Running Title: Trainability of the History Dependence of Force

Key Words: Fascicle, muscle, residual force enhancement, residual force depression, sarcomere, eccentric, concentric, uphill running, downhill running

Correspondence: Geoffrey A. Power PhD.

Neuromechanical Performance Research Laboratory

Department of Human Health and Nutritional Sciences

College of Biological Sciences

University of Guelph, Ontario, Canada

Telephone: 1-519-824-4120 x53752

Email: gapower@uoguelph.ca 
bioRxiv preprint doi: https://doi.org/10.1101/2020.03.31.018895; this version posted April 1, 2020. The copyright holder for this preprint (which was not certified by peer review) is the author/funder. All rights reserved. No reuse allowed without permission.

Trainability of the history dependence of force

\section{Summary Statement}

45

46 The addition and subtraction of serial sarcomeres induced by downhill and uphill running,

47 respectively, did not influence the magnitude of stretch-induced force enhancement and

48 shortening-induced force depression.

49 


\section{Trainability of the history dependence of force}

\section{Abstract}

51 The increase or decrease in isometric force following active muscle lengthening or shortening,

52 relative to a reference isometric contraction at the same muscle length and level of activation, are

53 referred to as residual force enhancement (rFE) and residual force depression (rFD), respectively.

54 The purpose of these experiments was to gain further mechanistic insight into the trainability of

$55 \mathrm{rFE}$ and $\mathrm{rFD}$, on the basis of serial sarcomere number (SSN) alterations to length-dependent

56 properties. Maximal rFE/rFD measures from the soleus and extensor digitorum longus (EDL) of

57 rats were compared after 4 weeks of uphill/downhill running and a no running control. Serial

58 sarcomere numbers adapted to the training: soleus serial sarcomere number was greater with

59 downhill compared to uphill running, while EDL demonstrated a trend towards more serial

60 sarcomeres for downhill compared to no running. In contrast, absolute and normalized rFE/rFD

61 did not differ across training groups for either muscle. As such, it appears that training-induced

62 SSN adaptations do not modify rFE/rFD at the whole-muscle level.

63

64 


\section{Trainability of the history dependence of force}

\section{Introduction}

The history dependence of force is an intrinsic property of skeletal muscle that has been investigated in individual sarcomeres (Joumaa and Herzog, 2010; Joumaa et al., 2008; Johnston et al., 2016; Leonard et al., 2010) to the whole-human level (Seiberl et al., 2015; Chapman et al., 2018; Chen et al. 2019), and is fundamental to a complete understanding of muscle contraction. Residual force enhancement (rFE) and residual force depression (rFD) are two history dependent force phenomena, characterized by increases and decreases in isometric force following active lengthening and shortening, respectively, in comparison to a reference isometric contraction at the same muscle length and level of activation (Abbott and Aubert, 1952; Chapman et al., 2018; Herzog, 2004; Rassier and Herzog, 2004b; Seiberl et al., 2015a). The magnitude of rFE is shown to be dependent on the amplitude of muscle lengthening and largely independent of lengthening velocity (Abbott and Aubert, 1952; Edman et al., 1978; Herzog and Leonard, 2002, 2005; Julian and Morgan, 1979; Fukutani et al., 2019b), while rFD appears to be strongly and positively related to the work (i.e., product of force $\times$ length change) of muscle shortening (Herzog and Leonard, 1997; Herzog et al., 2000; Lee et al., 2000). Suggested mechanisms include length-dependent contributions from non-contractile elements (i.e., titin) during lengthening for rFE (Herzog, 1998; Noble, 1992; Labeit et al., 2003; Nishikawa et al., 2012,; Joumaa and Herzog, 2014; Rode et al., 2009), and stress-induced angular actin deformations that impair cross bridge attachments during shortening for rFD (Joumaa et al., 2018; Maréchal and Plaghki, 1979). Given that rFE and rFD are likely to be intertwined in everyday movements (e.g., during stretch-shortening cycles), a means of maximizing rFE and/or minimizing rFD - the former being associated with increased neuromuscular economy (i.e., more force per unit of activation (Jones et al., 2016; Paquin and 


\section{Trainability of the history dependence of force}

87 Power, 2018) and reduced adenosine triphosphate usage (Joumaa and Herzog, 2013) - would be

88 desirable to improving motor efficiency.

While there have been recent investigations into the plasticity of the history dependence of

90 force in states of altered contractile capacity (Dargeviciute et al., 2013; Power et al., 2012a, 2012b,

91 2013, 2014a, 2014b; Ramsey et al., 2010; de Ruiter et al., 2000; de Ruiter and de Haan, 2003),

92 there is still little work looking into its trainability (Chen and Power, 2019; Siebert et al., 2016).

93 Prior studies have demonstrated contraction-type-dependent morphological adaptations with

94 training, whereby eccentric loading leads to increases in fascicle lengths/serial sarcomere numbers

95 (Butterfield and Herzog, 2005; Franchi et al., 2014; Lynn and Morgan, 1994; Lynn et al., 1998;

96 Reeves et al., 2009; Timmins et al., 2016) and concentric loading leads to decreases in fascicle

97 lengths/serial sarcomere numbers (Butterfield and Herzog, 2005; Lynn and Morgan, 1994; Lynn

98 et al., 1998, Timmins et al., 2016; Morais et al. 2019). Considering that rFE and rFD are length-

99 dependent properties, eccentric- and concentric-biased training could potentially modify rFE and

100 rFD through respective increases and decreases to the number of sarcomeres in series that modulate

101 the amplitude of sarcomere lengthening and shortening experienced for a given muscle length

102 change. A study by Chen and Power (2019) found that rFE (but not rFD) was differentially

103 increased by chronic (4-week) concentric resistance training, and decreased by chronic eccentric

104 resistance, owing to a likely combination of mechanical and neurological factors. While there was

105 ultrasound evidence of fascicle length adaptations, the data were obtained from a small subset of

106 the participants. As such, it remains to be elucidated: (i) what mechanical factors were responsible

107 for the alterations to history-dependent force properties, and (ii) whether these alterations would

108 persist after accounting for supposed serial sarcomere number differences. 


\section{Trainability of the history dependence of force} Therefore, the purpose of these experiments was to gain additional mechanistic insight into

110 the adaptability of the history dependence of force. We employed a model of uphill- and downhill-

111 running rats to allow for an isolated (in vitro) approach to investigate muscle contractile properties

112 and sarcomerogenesis. It was hypothesized that for an absolute muscle length change, rFE and

113 rFD would increase following concentric training and decrease following eccentric training, owing

114 to a respective reduction and addition of sarcomeres in series. However, should differences in

$115 \mathrm{rFE} / \mathrm{rFD}$ persist after normalizing for presumed serial sarcomere number discrepancies,

116 mechanisms other than lengthening/shortening amplitudes must contribute to the modifiability of

117 the history dependence of force following chronic incline/decline running. 


\section{Trainability of the history dependence of force}

118

119

120

121 for study, with approval from the Animal Care Committee of the University of Guelph. Rats were

122 housed in groups of three, with a maximum of two groups at any given time, and free fed a Teklad

123 global 18\% protein rodent diet (Envigo, Huntingdon, Cambs., UK) and water. After a week of

124 acclimation to the new housing conditions, each rat was familiarized with running and assigned to

125 one of three experimental groups: uphill running, downhill running, and sedentary control (i.e., no

126 running intervention). Following the 20 days of exercise, rats recovered for 72 hours before

127 sacrifice via $\mathrm{CO}_{2}$ asphyxiation followed by cervical dislocation, for experimental testing.

128

129

130

131

132

133

134

135

136

137

138

139

Training protocol: One week prior to training, rats were familiarized with treadmill running (on a $0^{\circ}$ grade). Rats in the exercise intervention groups (i.e., uphill/downhill running)

were run 5 days/wk (i.e., Mon-Fri) on an EXER 3/6 animal treadmill (Columbus Instruments, Columbus, $\mathrm{OH}$, USA) set to a $15^{\circ}$ incline or decline for 20 training days, over a 4-week period.

Training sessions lasted 15 minutes on the first day and the daily duration was increased by 5 min/day, up to the target 35 minutes (by the fifth training day) for the remainder of the training period. At the start of each training session, rats were first introduced to a walking speed of 10 $\mathrm{m} / \mathrm{min}$, which was gradually increased to the $16 \mathrm{~m} / \mathrm{min}$ target running speed, at a rate of $1 \mathrm{~m}$ every min. Rats were provided with 2 minutes of rest after each 5 minute bout. Running was encouraged with a firm tap or push from behind. Introduction into the training protocol was staggered so that the subsequent group could begin training once the previous group was halfway through their training period. 


\section{Trainability of the history dependence of force}

Experimental set up: Following sacrifice, the soleus (SOL) and extensor digitorum longus

142 (EDL) muscles were carefully harvested from the right hind limbs. Silk-braided sutures (USP 2-

1430 , metric 3) were tied along the musculotendinous junctions and mounted to the force-length

144 controller/transducers in the 806D Rat Apparatus (Aurora Scientific, Aurora, ON, Canada). The

145 muscles were bathed in a $\sim 25^{\circ} \mathrm{C}$ Tyrode solution (121 mM NaCl, $24 \mathrm{mM} \mathrm{NaHCO}, 5.5 \mathrm{mM}$ D-

146 Glucose, $5 \mathrm{mM} \mathrm{KCl}, 1.8 \mathrm{mM} \mathrm{CaCl}_{2}, 0.5 \mathrm{mM} \mathrm{MgCl}_{2}, 0.4 \mathrm{mM} \mathrm{NaH} \mathrm{PO}_{4}, 0.1 \mathrm{mM}$ EDTA) and

147 bubbled with a 95\% $\mathrm{O}_{2} / 5 \% \mathrm{CO}_{2}$ gas mixture (Praxair Canada Inc., Kitchener, ON, Canada) to a

$148 \mathrm{pH}$ of 7.4. A 701C High-Powered, Bi-Phase Stimulator (Aurora Scientific, Aurora, ON, Canada)

149 was used to evoke all contractions via two parallel platinum electrodes, submerged in the solution,

150 situated on either side of the muscle. Force, length, and stimulus trigger data were all sampled at

151 10,000 Hz with a 605A Dynamic Muscle Data Acquisition and Analysis System (Aurora

152 Scientific, Aurora, ON, Canada). All data were analyzed with the 615A Dynamic Muscle Control

153 and Analysis High Throughput (DMC/DMA-HT) software suite (Aurora Scientific, Aurora, ON,

154 Canada).

155 Experimental procedures: An experimental schematic is depicted in Fig. 1. Testing with

156 each muscle proceeded in order from Protocol A to G, with the order of fixed and relative protocols

157 being randomized within the rFD and rFE conditions. All rFD and rFE protocols consisted of an

$158 \mathrm{rFD} / \mathrm{rFE}$ trial and a reference isometric (ISO) trial. The rFD trials (in protocol C and D) always

159 preceded the ISO trials so as that the comparatively lower forces in the rFD trials could be

160 attributed to shortening-induced deficits, as opposed to fatigue. Likewise, the ISO trials (in

161 protocol E and F) always preceded the rFE trials so that the comparatively higher forces in the rFE

162 trials could be attributed to lengthening-induced enhancements, rather than fatigue. Contractions

163 in Protocols $\mathrm{C}$ to $\mathrm{G}$ were separated by $5 \mathrm{~min}$ intervals to minimize muscle fatigue. Prior to the 


\section{Trainability of the history dependence of force}

164 start of the protocols, the muscle was passively set to a taut length (measured tie-to-tie) that exerted

$165 \sim 0.075 \mathrm{~N}$ of resting tension, as a starting point for approximating optimal lengths for tetanic force 166 production $\left(\mathrm{L}_{0}\right)$.

167 Protocol A: Twitch current. Single 1.25-ms pulses were incremented by $0.5 \mathrm{~mA}$ (starting 168 from $1 \mathrm{~mA}$ ) until a current suitable to elicit peak twitch force was determined.

169 Protocol B: Optimal length. A maximal tetanic stimulation (pulse width: 0.3 ms, duration:

$1701 \mathrm{~s}$, frequency: $100 \mathrm{~Hz}$ ) was delivered before the muscle was passively shortened or stretched by

$1712 \mathrm{~mm}$ and a subsequent stimulation was delivered $1.5 \mathrm{~min}$ later. This was repeated until peak

172 tetanic force was obtained and the corresponding muscle length was measured. Once $\mathrm{L}_{\mathrm{o}}$ was

173 roughly determined, 1-mm and 0.5-mm increments were further used to more accurately establish

$174 \mathrm{~L}_{0}$.

Protocol C: Fixed rFD. The fixed rFD trial was comprised of 3 distinct yet continuous

176 phases: a 1-s pre-activation at $\mathrm{L}_{0}+2 \mathrm{~mm}$, a $-2 \mathrm{~mm} / \mathrm{s}$ isokinetic shortening to $\mathrm{L}_{0}-2 \mathrm{~mm}$, and a 3-s

177 isometric at $\mathrm{L}_{0}-2 \mathrm{~mm}$. The subsequent ISO.S trial consisted of a 6-s isometric at $\mathrm{L}_{0}-2 \mathrm{~mm}$.

179 phases: a 1-s pre-activation at $1.05 \mathrm{~L}_{0}$, a $-2 \mathrm{~mm} / \mathrm{s}$ isokinetic shortening to $0.95 \mathrm{~L}_{\mathrm{o}}$, and a 3-s isometric 180 at $0.95 \mathrm{~L}_{\mathrm{o}}$. The subsequent ISO.S trial consisted of a 6 -s isometric at $0.95 \mathrm{~L}_{0}$.

182 phases: a 1-s pre-activation at $\mathrm{L}_{0}-2 \mathrm{~mm}, \mathrm{a}+2 \mathrm{~mm} / \mathrm{s}$ isokinetic lengthening to $\mathrm{L}_{0}+2 \mathrm{~mm}$, and a 3-s

183 isometric at $\mathrm{L}_{\mathrm{o}}+2 \mathrm{~mm}$. The preceding ISO.L trial consisted of a $6-\mathrm{s}$ isometric at $\mathrm{L}_{\mathrm{o}}+2 \mathrm{~mm}$.

185 phases: a 1-s pre-activation at $0.95 \mathrm{~L}_{0}, \mathrm{a}+2 \mathrm{~mm} / \mathrm{s}$ isokinetic lengthening to $1.05 \mathrm{~L}_{0}$, and a 3-s

186 isometric at $1.05 \mathrm{~L}_{0}$. The preceding ISO.L trial consisted of a 6-s isometric at $1.05 \mathrm{~L}_{0}$. 


\section{Trainability of the history dependence of force}

Protocol G: Final muscle assessment. A final tetanic contraction was performed at $\mathrm{L}_{\mathrm{o}}$ to

188

189

190

191

192

193

194

195

196

197

198

199

200

201

202

203

204

205

206

207

208

209

assess decreases in isometric force-generating capacity.

Serial sarcomere number estimations: Following mechanical testing, muscles were removed from the bath, passively stretched to $\mathrm{L}_{0}$ (determined from protocol $\mathrm{B}$ ), tied to wooden sticks, and fixed in 10\% phosphate-buffered formalin for 48 hrs, rinsed with phosphate-buffered saline, and digested in 30\% nitric acid for at least 6 hrs to remove connective tissue and allow individual muscles fascicles to be teased out (Butterfield et al., 2005). Six fascicles were carefully obtained from deep and superficial regions of each muscle. Fascicle lengths (FL) were measured using 150 mm (resolution: 0.01 mm) electronic digital calipers (Marathon, Vaughan, ON, Canada).

Sarcomere length (SL) measurements were taken over six different regions across the length of each fascicle via laser diffraction (Coherent, Santa Clara, CA, USA) with a $5 \mathrm{~mW}$ diode laser (beam diameter: $25 \mu \mathrm{m}$, wavelength: $635 \mathrm{~nm}$ ) and custom LabVIEW program (Version 2011, National Instruments, Austin, TX, USA) (Lieber et al., 1984), for a total of 36 SL measurements per muscle. Serial sarcomere numbers (SSN) were calculated as:

\section{Serial sarcomere number $=$ fascicle length / average sarcomere length}

Data analyses: Steady-state isometric force values were calculated by subtracting passive force (resting baseline, pre-activation) from active force 2-s and 1-s following the end of the active length change for the SOL and EDL, respectively. Timing of 'steady-state' values were based on previous analysis of the rate of tension decay after stretch (Ramsey et al., 2010), in conjunction with visual confirmation from our own observations on the dissipation of force transients. Absolute rFE and rFD values (expressed in $\mathrm{N} / \mathrm{cm}^{2}$ ) were calculated as the increase and decrease 
210 in isometric force following active lengthening and shortening, respectively, relative to the ISO

211 contraction at the same time point into the active contraction (i.e., roughly 5-s and 4-s into the 6-s

212 contraction for the SOL and EDL, respectively). Whereas, normalized rFE and rFD values

213 (expressed as \%ISO) were calculated as the percent difference in steady-state isometric force,

214 relative to the ISO contraction, at the same time point. Similarly, passive FE (pFE) was calculated

215 as the absolute and percent increase in passive force following active lengthening, relative to

216 passive force (at rest, post-activation) of the ISO contraction. Peak eccentric force values were

217 taken as the highest value achieved during the active lengthening phase of the rFE trials. Work of

218 shortening values were calculated as the force-displacement integral for the active shortening

219 phase of the rFD trials. All force and work values were normalized to physiological cross-sectional

220 area (PCSA in $\mathrm{cm}^{2}$ ). PCSA was calculated as:

(1) PCSA = muscle mass $/$ (muscle density $\times$ normalized fascicle length)

Wherein, muscle density was assumed to be $1.112 \mathrm{~g} / \mathrm{cm}^{3}$ (Ward and Lieber, 2005), and normalized fascicle length was calculated as:

\section{(2) Normalized fascicle length $=$ fascicle length $\times$ (optimal sarcomere length $/$ measured sarcomere length)}

Wherein, optimal sarcomere length was assumed to be $2.72 \mu \mathrm{m}$ (based the average of all SL measurements)

231 inconsistencies in $\mathrm{L}_{0}$ that arose during the muscle fixation/digestion process.

A two-tailed paired $t$-test was used to compare force values between the history dependent

234 and ISO trials to ascertain the presence of rFE, rFD, and pFE. A two-way analysis of variance

235 (ANOVA) with a Holm-Sidak analysis for all pairwise comparisons was used to compare SSN,

$236 \mathrm{SL}, \mathrm{FL}, \mathrm{L}_{0}$, and $\mathrm{F}_{\mathrm{o}}$ values across muscles (SOL, EDL) and training groups (control, uphill,

237 downhill). A three-way ANOVA with a Holm-Sidak analysis for all pairwise comparisons was 
238 used to compare peak eccentric force, work of shortening, rFD, rFE, and pFE values across

239 muscles (SOL, EDL), training groups (control, uphill, downhill), and protocols (fixed, relative).

240 Significance was set to an $\alpha=0.05$. All data are reported as means \pm standard errors (SE). 
Trainability of the history dependence of force

\section{Results}

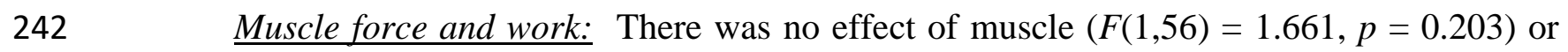

243 training $(F(1,56)=0.100, p=0.905)$ for isometric force at optimal length (Table 1$)$. There was a

244 main effect of muscle $(F(1,108)=62.378, p<0.001)$ for peak eccentric force, but no effect of

245 training $(F(1,108)=0.126, p=0.882)$ or protocol $(F(1,108)=0.895, p=0.346)$, whereby the SOL

246 produced 61\% higher eccentric force than the EDL (Table 1). Furthermore, there was a main effect

247 of muscle $(F(1,108)=19.043, p<0.001)$ and protocol $(F(1,108)=4.167, p=0.044)$ for work of

248 shortening, but no muscle $\times$ protocol interaction $(F(1,108)=1.988, p=0.161)$ or effect of training

$249(F(1,108)=0.0522, p=0.949)$, such that work performed during shortening was 37\% greater for

250 the SOL than EDL and 15\% greater for the fixed compared to relative protocol (Table 1).

251 In contrast, when normalized to PCSA, there was a main effect of muscle for specific (i.e.,

252 expressed in units $\left./ \mathrm{cm}^{2}\right)$ isometric force at optimal length $(F(1,56)=6.941, p=0.011)$, specific

253 peak eccentric force $(F(1,108)=65.911, p<0.001)$, and specific work of shortening $(F(1,108)=$

254 27.885, $p<0.001)$, but no effect of training $(p \geq 0.05)$ or protocol $(p \geq 0.05)$. Specifically, the

255 SOL exhibited 34\% higher specific isometric force, 96\% higher specific eccentric force, and

256 performed 65\% more specific work during shortening than the EDL across training groups and

257 protocols (Table 1).

Serial sarcomere numbers and muscle length measures: There was an interaction of

259 muscle $\times$ training $(F(2,371)=3.237, p=0.040)$ for SSN, in addition to main effects of muscle

$260(F(1,371)=119.567, p<0.001)$ and training $(F(2,371)=5.732, p=0.004)$. There was

261 significantly greater SSN for the SOL than EDL $(p=0.001)$ across training groups and protocols,

262 and the downhill group had a significantly greater SSN than both the uphill $(p=0.005)$ and control

263 ( $p=0.017)$ groups across muscles and protocols (Fig. 2). Within SOL, downhill running (95\% CI 
$264=6992.2,7237.1)$ resulted in a $6 \%$ greater SSN $(p=0.003)$ than uphill running $(95 \% \mathrm{CI}=6515.0$,

265 6895.0), while SSN for the EDL did not significantly differ across training groups ( $p \geq 0.05)$ (Fig.

266 2). Moreover, there was a main effect of muscle for fascicle length $(F(1,56)=39.358, p<0.001)$

267 and muscle length $(F(1,52)=604.518, p<0.001)$, but no effect of training $(p \geq 0.05)$ for either

268 dependent variable, such that the SOL had a 17\% shorter muscle but $13 \%$ longer fascicles

269 compared to the EDL (Table 2). In contrast, there was no effect of either muscle $(F(1,55)=0.734$,

$270 p=0.395)$ or training $(F(2,55)=0.182, p=0.834)$ on sarcomere lengths (Table 2$)$.

Residual force depression (Fig. 3A): There was a main effect of muscle $(F(1,108)=$

272 11.892, $p<0.001$ ) for absolute rFD (expressed in $\mathrm{N} / \mathrm{cm}^{2}$ ), but no effect of training $(F(2,108)=$

$2730.440, p=0.645)$ or protocol $(F(1,108)=2.793, p=0.098)$. Absolute rFD was present across all

274 training groups and protocols $(p<0.05)$ for both muscles, with $45 \%$ greater absolute rFD for the

275 SOL than EDL (Fig. 3B). Absolute rFD was positively and linearly associated with specific work

276 of shortening (Fig. 3D) for the SOL $\left(\mathrm{R}^{2}=0.71 ; F(1,29)=70.292, p<0.001\right)$ and $\operatorname{EDL}\left(\mathrm{R}^{2}=0.61\right.$;

$277 F(1,29)=45.362, p<0.001)$. In contrast, there was no significant relationship between absolute

278 rFD and SSN (SOL: $\mathrm{R}^{2}=0.051, p=0.228$; EDL: $\left.\mathrm{R}^{2}=0.107, p=0.073\right)$, fascicle length (SOL: $\mathrm{R}^{2}$

$279=0.012, p=0.565 ;$ EDL: $\mathrm{R}^{2}=0.104, p=0.077$ ), or muscle length $\left(\mathrm{SOL}: \mathrm{R}^{2}=0.0004, p=0.914\right.$

280 EDL: $\mathrm{R}^{2}=0.113, p=0.074$ ) for either muscle.

Likewise, there was a main effect of muscle $(F(1,108)=67.200, p<0.001)$ for normalized

282 rFD (expressed as \%ISO.S), but no effect of training $(F(2,108)=0.295, p=0.745$ ) or protocol

$283(F(1,108)=1.526, p=0.219)$. Normalized rFD was present across all training groups and

284 protocols $(p<0.05)$ for both muscles, with $2.1 \times$ greater normalized rFD for the EDL than SOL

285 (Fig. 3C). There was no significant relationship between normalized rFD and specific work of

286 shortening (SOL: $\mathrm{R}^{2}=0.028, p=0.364$; EDL: $\left.\mathrm{R}^{2}=0.026, p=0.386\right), \mathrm{SSN}\left(\mathrm{SOL}: \mathrm{R}^{2}=0.087, p=\right.$ 
287

288

289

290

291

292

293

294

295

296

297

298

299

300

301

302

303

304

305

306

307

308

0.113; EDL: $\left.\mathrm{R}^{2}=0.001, p=0.845\right)$, fascicle length (SOL: $\mathrm{R}^{2}=0.051, p=0.223$; EDL: $\mathrm{R}^{2}=0.001$, $p=0.845$ ), or muscle length (SOL: $\mathrm{R}^{2}=0.001, p=0.851$; $\mathrm{EDL}: \mathrm{R}^{2}=0.113, p=0.074$ ) for either muscle.

Residual force enhancement (Fig 4A): There was a main effect of muscle $(F(1,08)=$ 30.138, $p<0.001)$ and protocol $(F(1,2)=0.0736, p=0.004)$ for absolute $\mathrm{rFE}\left(\right.$ expressed in $\left.\mathrm{N} / \mathrm{cm}^{2}\right)$, but no significant muscle $\times$ protocol interaction $(F(2,108)=3.908, p=0.051)$ or effect of training $(F(2,108)=0.0736, p=0.929)$. Absolute rFE was present across all training groups and protocols $(p<0.05)$ for both muscles (Fig. 4B), wherein absolute rFE was 2.3x greater for the SOL than EDL across training groups and protocols, and 56\% greater for the fixed compared to relative protocol across muscles and training groups. Absolute rFE was positively and linearly associated with specific eccentric force (Fig. 4D) for the SOL $\left(\mathrm{R}^{2}=0.57 ; F(1,29)=39.108, p<0.001\right)$ and EDL $\left(\mathrm{R}^{2}=0.72 ; F(1,29)=76.100, p<0.001\right)$. Whereas, there was no significant relationship between absolute rFE and SSN (SOL: $\mathrm{R}^{2}=0.0004, p=0.913$; EDL: $\mathrm{R}^{2}=0.086, p=0.110$ ), fascicle length (SOL: $\mathrm{R}^{2}=0.004, p=0.744$; EDL: $\mathrm{R}^{2}=0.079, p=0.127$ ), or muscle length (SOL: $\mathrm{R}^{2}=$ $0.012, p=0.573$; EDL: $\mathrm{R}^{2}=0.001, p=0.856$ ) for either muscle.

In contrast, there was a main effect of protocol $(F(1,108)=7.014, p=0.009)$ for normalized rFE (expressed as \%ISO.L), but no effect of muscle $(F(1,108)=0.759, p=0.386$ ) or training $(F(2,108)=0.805, p=0.450)$. Normalized rFE was present across all training groups $(p<0.05)$ for both muscles, with $43 \%$ greater normalized rFE for the fixed compared to relative protocol (Fig. 4C). There was no significant relationship between normalized rFE and specific eccentric force $\left(\mathrm{SOL}: \mathrm{R}^{2}=0.026 ; F(1,29)=0.852, p=0.364 ; \mathrm{EDL}: \mathrm{R}^{2}=0.022 ; F(1,29)=0.641, p=0.430\right)$, SSN (SOL: $\mathrm{R}^{2}=0.054, p=0.216 ;$ EDL: $\mathrm{R}^{2}=0.039, p=0.284$ ), fascicle length (SOL: $\mathrm{R}^{2}=0.040$ 


\section{Trainability of the history dependence of force}

309

310

311

312

313

314

315

316

317

318

319

320

321

322

323

324

325

326

327

328

329

330

331

$\left.p=0.280 ; \mathrm{EDL}: \mathrm{R}^{2}=0.040, p=0.280\right)$, or muscle length $\left(\mathrm{SOL}: \mathrm{R}^{2}=0.004, p=0.747 ; \mathrm{EDL}: \mathrm{R}^{2}\right.$ $=0.006, p=0.689$ ) for either muscle.

Passive force enhancement: There was a main effect of muscle $(F(1,107)=24.172, p<$ 0.001 ) for absolute pFE (expressed in $\left.\mathrm{N} / \mathrm{cm}^{2}\right)$, but no effect of training $(F(1,107)=1.151, p=$ 0.320 ) or protocol $(F(1,107)=1.1491, p=0.225)$. Absolute $\mathrm{pFE}$ was present across all training groups and protocols $(p<0.05)$ for both muscles, with $2.2 \times$ greater absolute pFE for the SOL than EDL (Fig. 5A). Absolute pFE was positively and linearly associated with absolute rFE (Fig. 5C) for the SOL $\left(\mathrm{R}^{2}=0.41 ; F(1,29)=19.897, p<0.001\right)$ and $\operatorname{EDL}\left(\mathrm{R}^{2}=0.69 ; F(1,29)=65.279, p<\right.$ $0.001)$, amounting to $29 \%\left(0.71 \pm 0.10 \mathrm{~N} / \mathrm{cm}^{2}\right)$ and $37 \%\left(0.30 \pm 0.047 \mathrm{~N} / \mathrm{cm}^{2}\right)$ of the absolute rFE observed for the SOL and EDL, respectively. Furthermore, absolute pFE was positively and linearly associated with specific eccentric force (Fig. 5D) for the SOL $\left(\mathrm{R}^{2}=0.49 ; F(1,29)=28.212\right.$, $p<0.001)$ and EDL $\left(\mathrm{R}^{2}=0.65 ; F(1,29)=53.236, p<0.001\right)$. Meanwhile, for the SOL, there was no significant relationship between absolute pFE and SSN $\left(\mathrm{R}^{2}=0.010, p=0.596\right)$, fascicle length $\left(\mathrm{R}^{2}=0.032, p=0.341\right)$, or muscle length $\left(\mathrm{R}^{2}=0.124, p=0.061\right)$. Whereas for the EDL, there was weak but significant relationship between absolute pFE vs. SSN $\left(\mathrm{R}^{2}=0.158 ; F(1,29)=5.458\right.$; $p=0.027)$ and absolute pFE vs. fascicle length $\left(\mathrm{R}^{2}=0.150 ; \mathrm{F}(1,29)=5.124, p=0.031\right)$, but not between absolute pFE and muscle length $\left(\mathrm{R}^{2}=0.003, p=0.768\right)$.

Similarly, there was a main effect of muscle $(F(1,107)=31.979, p<0.001)$ for normalized pFE (expressed as \%ISO.L), but no effect of training $(F(1,107)=1.152, p=0.320$ ) or protocol $(F(1,107)=0.149, p=0.700)$. Normalized $p F E$ was present across all training groups and protocols $(p<0.05)$ for both muscles, with $3.3 \times$ greater normalized pFE for the SOL than EDL (Fig. 5B). There was no significant relationship between normalized pFE and normalized rFE for the SOL $\left(\mathrm{R}^{2}=0.028, p=0.367\right)$ but a significant, albeit weak, relationship for the EDL $\left(\mathrm{R}^{2}=\right.$ 
332 0.152; $F(1,29)=5.195, p=0.030$ ). Additionally, there was a weak but significant relationship

333 between normalized pFE and specific eccentric force for the SOL $\left(\mathrm{R}^{2}=0.180 ; F(1,29)=6.377, p\right.$

$334=0.017)$ and $\operatorname{EDL}\left(\mathrm{R}^{2}=0.223 ; F(1,29)=8.300, p=0.007\right)$. Finally, there was no significant

335 relationship between normalized pFE and SSN (SOL: $\mathrm{R}^{2}=0.0003, p=0.927$; EDL: $\mathrm{R}^{2}=0.050, p$

$336=0.225)$, fascicle length (SOL: $\mathrm{R}^{2}=3.644 \times 10^{-5}, p=0.974$; EDL: $\mathrm{R}^{2}=0.047, p=0.240$ ), or

337 muscle length (SOL: $\mathrm{R}^{2}=0.036, p=0.327$; EDL: $\mathrm{R}^{2}=0.009, p=0.617$ ) for either muscle. 


\section{Trainability of the history dependence of force}

339

340

341

342

343

344

345

346

347

348

\section{Discussion}

The purpose of these experiments were to determine whether the history dependence of force could be modified by contraction-type-dependent SSN adaptations. Serial sarcomere number and history-dependent forces for the SOL and EDL muscles of rats were compared across training groups following 4-weeks of uphill and downhill running. In accordance with our hypothesis, there was a greater SSN for the SOL with downhill compared to uphill running, and a trend towards a greater SSN for the EDL with downhill running in comparison to the control group. While SSN appeared to differ with training, History-dependent forces were not similarly affected. Together, these results indicate that training-induced SSN adaptations do not modify wholemuscle in-vitro measures of rFD, rFE, and pFE in the lower hind limb muscles of rats.

Serial sarcomere number adaptations: In the present study, SSN (Fig. 2) were consistent with the 7500 count for the VL (Butterfield et al., 2005), with values across muscles ranging from 3400 for the VI of rats (Lynn and Morgan, 1994; Lynn et al., 1998; Butterfield et al., 2005) to 21,000 for the TA of rabbits (Butterfield and Herzog, 2006). While our measured $2.72 \mu \mathrm{m}$ SL average was towards the higher end of the typical 2.4-2.8 $\mu \mathrm{m}$ range (Stephenson and Williams, 1982), values from this study were obtained from muscles (fascicles) passively fixed at their optimal length for force production, as opposed to given joint angle or skinned fibre. As a result, the compliance of the in series connective tissues would result in a shortening of sarcomeres upon activation and lengthening upon deactivation, and subsequently, an overestimation of SL when measured at a passive length.

In line with previous findings (Lynn and Morgan, 1994; Lynn et al., 1998; Butterfield et al., 2005; Morais et al. 2019), downhill running resulted in 6\% greater SSN for the SOL when compared to uphill running, but not a sedentary control group. Moreover, while SSN were not 


\section{Trainability of the history dependence of force}

362 significantly different across training groups for the EDL, there was a trend $(p=0.056)$ towards

$3634 \%$ greater SSN for the downhill vs. control group, indicating a potential stimulus for

364 sarcomerogenesis with downhill running. Given that strain dynamics were not measured during

365 training, insight into the actions of the SOL and EDL during uphill and downhill running have to

366 be inferred based on previous observations. In a similar treadmill training program as the current

367 study, Morais et al (2019) showed, in mice, concentric training led to a decrease in SSN for the

368 VL and TA while eccentric training had no effect. Additionally, there was no training induced

369 sarcomerogenesis effect for the GM and VM. However, when inferring SSN adaptations it is

370 important to consider the specific muscle fibre excursion during locomotion (Hu et al. 2017). It

371 is possible those muscles which did not show sarcomerogenesis did not experience a minimal

372 threshold to warrant adaptations.

373 During rat locomotion, soleus activity begins just prior to foot contact and ends

374 immediately before foot lift-off, whereas EDL activity begins just after foot lift-off and ends

375 immediately after foot contact (Nicolopoulos-Stournaras and Iles, 1984). This would imply that

376 the SOL is loaded eccentrically during downhill running and concentrically during uphill running,

377 which would support the findings of increased SSN with eccentric training and decreased SSN

378 with concentric training (Butterfield et al., 2005; Butterfield and Herzog, 2006). While not

379 statistically significant, the greater apparent number of sarcomeres for both training groups in

380 comparison to the sedentary control suggests that the EDL performed eccentric contractions during

381 downhill and uphill running, which may be explained by eccentric braking actions at the onset of

382 foot contact.

Strength measures: Perhaps contrary to expectations, training did not alter isometric

384 forces, eccentric forces, and work of shortening for either muscle. However, unlike the strength- 


\section{Trainability of the history dependence of force}

385

386

387

388

389

390

391

392

393

394

395

396

397

398

399

400

401

402

403

404

405

406

407

oriented resistance training employed by Chen and Power (2019), the main goal of our incline/decline-running model was to induce differential SSN adaptations, which has previously been demonstrated for the quadriceps muscles of rats (Lynn and Morgan, 1994; Lynn et al., 1998; Butterfield et al., 2005) and is partially supported by the present findings for the lower hind limb muscles. Yet, SSN increases were not mirrored by increases in muscle length (Table 2) or force/work performed (Table 1). However, architectural adaptations at the level of the fascicle do not always translate to the whole muscle (Sharifnezhad et al., 2014), owing to factors such as pennation angles and connective tissue compliance, which mediate the transfer of forces across functional scales. Furthermore, there is the ever-present issue of 'testing specificity'; assuming that neuromuscular adaptations are geared towards specific environmental perturbations, testing the muscle under different physiological/contractile conditions (i.e., in vitro) could likely wash out potential in-vivo responses (e.g., strain of specific muscle fibre regions during locomotion). Additionally, while SSN were significantly higher for the SOL following downhill vs. uphill running, from a functional standpoint, a $6 \%$ increase might not be sufficient to elicit prominent length-dependent changes in muscle force, as evidenced by the current results.

With respect to the rFE and rFD contractions, during a 4 mm muscle length change (i.e., in the fixed protocols) the $6 \%$ SSN difference for the SOL would roughly equate to an average sarcomere length change of $0.563 \mu \mathrm{m}$ for the downhill group and $0.596 \mu \mathrm{m}$ for the uphill group. However, this $0.033 \mu \mathrm{m}$ difference in sarcomere length does not account for confounding variables such as pennation angle, curvature of the fascicle, connective tissue compliance, and sarcomere length non-uniformities. Therefore, irrespective of whether SSN adaptations also took place for the EDL, it is likely that SSN differences would not be large enough to promote length-dependent differences in force at the whole-muscle level. While it could be argued that longer training 


\section{Trainability of the history dependence of force}

408

409

410

411

412

413

414

415

416

417

418

419

420

421

422

423

424

425 for normalized rFD. The discrepancy between relative and absolute values of rFD in the present

426 study is most likely due to reduced contractile capacity (i.e., possibly fatigue) of the predominantly

427 fast-twitch EDL muscle (Brooks and Faulkner, 1991), as evidenced by the comparatively lower

428 overall strength measures (Table 1). Given that the underlying factor contributing to rFD is a

429 stress-induced impairment of cross bridge attachments during active shortening, it is not surprising

430 then that a reduced capacity to perform work during shortening would result in lower absolute 


\section{Trainability of the history dependence of force}

431 values of rFD (Fig. 3D). However, after accounting for differences in isometric strength (i.e.,

432 lower contractile capacity), normalized rFD was greater for the EDL, suggesting greater intrinsic

433 rFD for fast-twitch muscles, owing to distinct force-velocity relationships that facilitate the

434 capacity to more produce work at a given shortening velocity, as compared with slow-twitch 435 muscles (Joumaa et al., 2015; Pinnell et al. 2019).

436 Contrary to our hypothesis, absolute and normalized rFD values were not different

437 following uphill and downhill training for either muscle, despite differences in SSN for the SOL,

438 and possibly, EDL. As a result, it is possible that SSN adaptations were not prominent enough or

439 specifically geared to evoke functional length-dependent differences under our in-vitro contractile

440 conditions. The lack of training-induced alterations to rFD in the present study is consistent with

441 findings from Chen and Power (2019), suggesting that neither SSN adaptations (in the present

442 study) nor potential neurological adaptations (in Chen and Power, 2019) serve to modify rFD at

443 the whole-muscle level.

$444 \quad$ Residual force enhancement: In line with the 8-52\% rFE previously reported in vitro

445 (Abbott and Aubert, 1952; Edman et al., 1982; Meijer, 2002; Pun et al., 2010; Rassier and Pavlov,

446 2012; Sugi and Tsuchiya, 1988), steady-state isometric force values following active lengthening

447 were $24 \%$ and $27 \%$ higher than the ISO for the SOL and EDL, respectively. Absolute rFE was

$4482.3 \times$ greater for the SOL than EDL while normalized rFE was not significantly different between

449 muscles, contrary to observations from Ramsey et al. (2010) of 55\% greater normalized rFE for

450 the EDL compared to SOL muscle of rats. In argument against reports that absolute rFE is

451 preserved in conditions of reduced contractile force (Fukutani and Herzog, 2018b; Rassier and

452 Herzog, 2004a), we show that absolute rFE is strongly and positively associated with the force 


\section{Trainability of the history dependence of force}

453 established during the active lengthening phase of the rFE contraction (Fig. 4D), suggesting a

454 strength-dependent component of rFE.

Although rFE is typically attributed to non-contractile elements (i.e., titin), contractile

456 contributions (e.g., decreased cross bridge detachment) cannot be discounted (Rassier and Herzog,

457 2004a; Lee et al., 2007; Pinnell et al., 2019). Furthermore, cross bridge cycling is necessary for

458 the engagement of both contractile and non-contractile elements involved in $\mathrm{rFE}$ and $\mathrm{pFE}$ 459 (Fukutani et al., 2019b, Lee et al., 2007; Pinnell et al., 2019; Rassier and Herzog, 2005; Rassier et

460 al., 2003; Herzog and Leonard, 2002; Rassier and Herzog, 2004a). Therefore, with reduced

461 contractile capacity, cross bridge kinetics would become impaired, leading to hypothetical

462 decreases in cross-bridge- and/or titin-mediated force enhancement. As such, the lower absolute

463 rFE for the EDL (relative to the SOL) is attributed to a lower contractile capacity of muscle

464 (Brooks and Faulkner, 1991), as evidenced by the comparatively lower overall strength measures

465 for the EDL (Table 2). Conversely, after accounting for differences in isometric force (i.e.,

466 strength/weakness), normalized rFE was not different between the SOL and EDL, suggesting a

467 lack of intrinsic difference for rFE between slow-twitch and fast-twitch muscles. While in contrast

468 to Ramsey et al. (2010), and reports of stiffer titin isoforms for predominantly fast-twitch muscles

469 (Horowits et al., 1986; Prado et al., 2005), observations from Fukutani et al. (2018b) of

470 similar/greater absolute and normalized rFE values for the soleus (94.5\% type I fibres) compared

471 to psoas (100\% type IIa fibres) muscle of rabbits (Prado et al., 2005) are in line with our findings.

472 Contrary to our hypothesis, absolute and normalized rFE values were not different

473 following uphill and downhill training for either muscle, despite differences in SSN for the SOL,

474 and possibly, EDL. In the same context as rFD, it is possible that the SSN adaptations were not

475 prominent enough or specifically geared to evoke functional length-dependent differences under 


\section{Trainability of the history dependence of force}

476 our in-vitro contractile conditions. The lack of training-induced alterations to rFE in the present

477 study is in agreement with Siebert et al. (2015), but in contrast to findings from Chen and Power

478 (2019), which reported increases to rFE with concentric training and decreases to rFE with

479 eccentric training. Discrepancies between this study and the one by Chen and Power (2019) may

480 be due to differences in training protocols, neurological components, and study designs. Training

481 in Chen and Power (2019) was predominantly load-focused, with in-vivo torque measurements

482 performed in the presence of an intact neurological system, the latter of which is primarily

483 responsible for strength adaptations (e.g., increased motor unit activation/decreased neural

484 inhibition) within the early phases (i.e., first 3-4 weeks) of resistance training (Douglas et al., 2017;

485 Hahn, 2018). In contrast, the current training protocol involved a large aerobic component, with

486 in-vitro force measurements performed independent of neurological contributions. Additionally,

487 Chen and Power (2019) looked at within-individual comparisons (pre- and post-training), whereas,

488 this study and the one by Siebert et al. (2016) compared values in a cross-sectional manner, which

489 could have masked more minute responses. Ultimately though, the results suggest that length-

490 dependent alterations to history-dependent properties via SSN adaptations do not serve to modify

491 rFE (or rFD) at the whole-muscle level.

492 Passive force enhancement: In line with the $\sim 3-54 \%$ pFE previously observed in the

493 literature (Herzog and Leonard, 2002; 2005; Lee and Herzog, 2002; Lee et al., 2007), resting forces

494 following active lengthening were 37\% and 11\% higher than those from the ISO for the SOL and

495 EDL, respectively. Additionally, pFE contributed to 29\% and 37\% of the rFE for the SOL and

496 EDL, respectively, which is consistent with the $\sim 8-84 \%$ pFE-to-rFE ratio reported in the literature

497 (Herzog and Leonard, 2002; 2005; Lee and Herzog, 2002; Lee et al., 2007), and highlights a

498 common history dependent mechanism. Considering that pFE is strongly and positively associated 

with rFE (Fig. 5C), the simultaneously greater absolute and normalized pFE values for the SOL

500 compared to EDL is in contrast to the greater absolute but similar normalized rFE observed.

501 Disparity between normalized values of rFE and pFE is most likely owing to differences between

502 the isometric reference contractions, wherein active force (for rFE) is diminished but passive 503 tension (for pFE) is preserved with lower contractile capacity. In other words, strength-related 504 impairments to cross bridge cycling would lead to concurrent decreases in absolute rFE and pFE 505 for the EDL (as illustrated by the strong, positive relationships to eccentric force in Fig. 4D and 506 Fig. 5D), but normalization to similar resting forces would not amplify pFE values like smaller

507 ISO forces would to rFE. As a consequence, comparisons cannot be made regarding intrinsic 508 differences in pFE between the SOL and EDL from this study.

509 Although absolute values of pFE were not associated with SSN or fascicle lengths for the 510 SOL, there were significant, albeit weak, relationships for the EDL, which highlight the length511 dependence of pFE for fast-twitch muscles (shown to possess stiffer titin isoforms). Yet, the fact

512 that absolute and normalized pFE values were not different following uphill and downhill training

513 for either muscle in the present study only further reinforces the idea that SSN adaptations were

514 ineffective at inducing in-vitro, length-dependent alterations to history-dependent forces at the 515 whole-muscle level.

516 This is the first study to demonstrate SSN differences for the soleus, and possibly EDL, 517 muscle of uphill- and downhill-running rats, which had previously only been reported for the VI, 518 VL, and TA, and recently the VL: and TA of mice (Morais et al. 2019). Consequently, it appears 519 that rFD, rFE, and pFE are not differentially modifiable at the whole-muscle level, on the basis of 520 training-induced alterations to SSN, as a means of altering sarcomere shortening/lengthening 521 amplitudes. However, these findings highlight the relevance of reporting both absolute and 


\section{Trainability of the history dependence of force}

522 relative history-dependent force values, which suggest that absolute values of rFD, rFE, and pFE

523 are not maintained during conditions of reduced contractile force, most likely owing to impaired

524 cross bridge engagement. In addition to furthering our understanding of muscle contractions, from

525 a practical standpoint, increases to rFE/decreases to rFD could serve to improve the neuromuscular

526 economy of functional movements, particularly during SSCs, wherein rFE appears to attenuate

527 rFD in an amplitude-dependent manner. As such, increases to rFE could benefit force production

528 during both lengthening- and shortening-induced conditions. Considering how intrinsic cross

529 bridge dynamics are to history dependent force properties (and vice versa), future studies

530 investigating the trainability of the history dependence of force should look to improve the

531 contractile capability of muscle through strength-focused training protocols. 


\section{Trainability of the history dependence of force}

\section{Acknowledgments}

534

535

536

537

538

539

540

541

542

543

\section{4}

545

546

547

This project was supported by the Natural Sciences and Engineering Research Council of Canada (NSERC). Infrastructure was provided by the University of Guelph start-up funding. No conflicts of interest, financial or otherwise, are declared by the authors.

\section{Disclosure statement}

No conflicts of interest, financial or otherwise, are declared by the authors.

\section{Ethics statement}

All procedures were approved by the Animal Care Committee of the University of Guelph.

\section{Data accessibility}

Individual values of all supporting data are available upon request.

\section{Grants}

This project was supported by the Natural Sciences and Engineering Research Council of Canada (NSERC). Infrastructure was provided by the University of Guelph start-up funding.

\section{Author contributions}

All authors contributed equally 
Trainability of the history dependence of force

\section{References}

1. Abbott BC and Aubert XM. The force exerted by active striated muscle during and after change of length. J Physiol 117: 77-86, 1952.

2. Brooks SV and Faulkner JA. Forces and powers of slow and fast skeletal muscles in mice during repeated contractions. J Physiol 436: 701-710, 1991.

3. Butterfield TA and Herzog W. The magnitude of muscle strain does not influence serial sarcomere number adaptations following eccentric exercise. Pflugers Arch 451: 688-700, 2006.

4. Butterfield TA, Leonard TR, and Herzog W. Differential serial sarcomere number adaptations in the knee extensor muscles of rats is contraction type dependent. $J$ Appl Physiol 99: 1352-1358, 2005.

5. Chapman N, Whitting J, Broadbent S, Crowley-Mchattan Z, and Meir R. Residual force enhancement in humans: a systematic review. J Appl Biomech 34: 240-248, 2018.

6. Chen $\mathbf{J}$ and Power GA. Modifiability of the history dependence of force through eccentric and concentric biased resistance training. J Appl Physiol 126: 647-657, 2019.

7. Dalton BH, Contento VS, and Power GA. Residual force enhancement during submaximal and maximal effort contractions of the plantar flexors across knee angle. $J$ Biomech 78: 70-76, 2018.

8. Dargeviciute G, Masiulis N, Kamandulis S, Skurvydas A, and Westerblad H. Residual force depression following muscle shortening is exaggerated by prior eccentric drop jump exercise. J Appl Physiol 115: 1191-1195, 2013.

9. De Ruiter CJ and de Haan A. Shortening-induced depression of voluntary force in unfatigued and fatigued human adductor pollicis muscle. J Appl Physiol 94: 69-74, 2003.

10. De Ruiter CJ, Didden WJ, Jones DA, and Haan AD. The force-velocity relationship of human adductor pollicis muscle during stretch and the effects of fatigue. J Physiol 526: 671-681, 2000.

11. Douglas J, Pearson S, Ross A, and McGuigan M. Chronic adaptations to eccentric training: a systematic review. Sports Med 47: 917-941, 2017.

12. Edman KAP, Elzinga G, and Noble MIM. Enhancement of mechanical performance by stretch during tetanic contractions of vertebrae skeletal muscle fibres. J Physiol 281: 139155, 1978.

13. Edman KAP, Elzinga G, and Noble MIM. Residual force enhancement after stretch of contracting frog single muscle fibers. J Gen Physiol 80: 769-784, 1982.

14. Edman KAP. Mechanical deactivation induced by active shortening in isolated muscle fibres of the frog. $J$ Physiol 241: 255-275, 1975.

15. Eng CM, Smallwood LH, Rainiero MP, Lahey M, Ward SR, and Lieber RL. Scaling of muscle architecture and fiber types in the rat hindlimb. J Exp Biol 211: 2335-2345, 2008.

16. Franchi MV, Atherton PJ, Reeves ND, Flück M, Williams J, Mitchell WK, Selby A, Beltran Vallis RM, and Narici MV. Architectural, functional and molecular responses to concentric and eccentric loading in human skeletal muscle. Acta Physiol (Oxf) 210: 642654, 2014.

17. Franchi MV, Reeves ND, and Narici MV. Skeletal muscle remodeling in response to eccentric vs. concentric loading: morphological, molecular, and metabolic adaptations. Front Physiol 8: 447, 2017. 


\section{Trainability of the history dependence of force}

603

604

605

606

607

608

609

610

611

612

613

614

615

616

617

618

619

620

621

622

623

624

625

626

627

628

629

630

631

632

633

634

635

636

637

638

639

640

641

642

643

644

645

646

647

18. Fukutani A and Herzog W. Residual force enhancement is preserved for conditions of reduced contractile force. Med Sci Sports Exerc 50: 1186-1191, $2018 \mathrm{~b}$.

19. Fukutani A, Leonard T, and Herzog W. Does stretching velocity affect residual force enhancement? J Biomech 89: 143-147, 2019 b.

20. Hahn D. Stretching the limits of maximal voluntary eccentric force production in vivo. $J$ Sport Health Sci 7: 275-281, 2018.

21. Herzog W and Leonard TR. Depression of cat soleus forces following isokinetic shortening. J Biomech 30: 865-872, 1997.

22. Herzog $\mathbf{W}$ and Leonard TR. Force enhancement following stretching of skeletal muscle: a new mechanism. J Exp Biol 205: 1275-1283, 2002.

23. Herzog $\mathbf{W}$ and Leonard TR. The history dependence of force production in mammalian skeletal muscle following stretch-shortening and shortening-stretch cycles. J Biomech 33: 531-542, 2000.

24. Herzog $\mathbf{W}$ and Leonard TR. The role of passive structures in force enhancement of skeletal muscles following active stretch. J Biomech 38: 409-415, 2005.

25. Herzog W. History dependence of skeletal muscle force production: implications for movement control. Hum Mov Sci 23: 591-604, 2004.

26. Herzog W, Leonard TR, and Wu JZ. Force depression following skeletal muscle shortening is long lasting. $J$ Biomech 31: 1163-1168, 1998.

27. Herzog W, Leonard TR, and Wu JZ. The relationship between force depression following shortening and mechanical work in skeletal muscle. $J$ Biomech 33: 659-668, 2000.

28. Herzog W. Passive force enhancement in striated muscle. J Appl Physiol 126: 1782-1789, 2019.

29. Horowits R, Kempner E, Bisher M, and Podolsky R. A physiological role for titin and nebulin in skeletal muscle. Nature 323: 160-164, 1986.

30. Hu X, Charles JP, Akay T, Hutchinson JR, Blemker SS. Are mice good models for human neuromuscular disease? Comparing muscle excursions in walking between mice and humans. Skeletal muscle. 7(1):26, 2017

31. Jones AA, Power GA, and Herzog W. History dependence of the electromyogram: Implications for isometric steady-state EMG parameters following a lengthening or shortening contraction. J Electromyogr Kinesiol 27: 30-38, 2016.

32. Joumaa $\mathbf{V}$ and Herzog W. Calcium sensitivity of residual force enhancement in rabbit skinned fibers. Am J Physiol Cell Physiol 307: 395-401, 2014.

33. Joumaa $\mathbf{V}$ and Herzog $\mathbf{W}$. Energy cost of force production is reduced after active stretch in skinned muscle fibres. J Biomech 46: 1135-1139, 2013.

34. Joumaa V and Herzog W. Force depression in single myofibrils. J Appl Physiol 108: 356362, 2010.

35. Joumaa V, Leonard TR, and Herzog W. Residual force enhancement in myofibrils and sarcomeres. Proc Biol Sci 275: 1411-1419, 2008.

36. Joumaa V, MacIntosh BR, Herzog W. New insights into force depression in skeletal muscle. J Exp Biol 215: 2135-2140, 2012.

37. Joumaa V, Power GA, Hisey B, Caicedo A, Stutz J, and Herzog W. Effects of fiber type on force depression after active shortening in skeletal muscle. J Biomech 48: 16871692, 2015. 


\section{Trainability of the history dependence of force}

38. Joumaa V, Smith IC, Fakutani A, Leonard T, Ma W, Irving T, and Herzog W. Evidence for actin filament structural changes after active shortening in skinned muscle bundles. Biophys J 114: 135a, 2018.

39. Julian FJ and Morgan DL. The effect of tension on non-uniform distribution of length changes applied to frog muscle fibres. J Physiol 293: 379-392, 1979.

40. Johnston K, Jinha A, and Herzog W. The role of sarcomere length non-uniformities in residual force enhancement of skeletal muscle myofibrils. $R$ Soc Open Sci 3: 150657, 2016.

41. Labeit D, Watanabe K, Witt C, Fujita H, Wu Y, Lahmers S, Funck T, Labeit S, and Granzier H. Calcium-dependent molecular spring elements in the giant protein titin. Proc Natl Acad Sci U S A 100: 13716-13721, 2003.

42. Lee EJ, Joumaa V, and Herzog W. New insights into the passive force enhancement in skeletal muscles. J Biomech 40: 719-727, 2007.

43. Lee HD and Herzog W. Force enhancement following muscle stretch of electrically stimulated and voluntarily activated human adductor pollicis. J Physiol 545: 321-330, 2002.

44. Lee HD, Suter E, and Herzog W. Effects of speed and distance of muscle shortening on force depression during voluntary contractions. J Biomech 33: 917-923, 2000.

45. Leonard TR, Duvall M, and Herzog W. Force enhancement following stretch in a single sarcomere. Am J Physiol Cell Physiol 299: C1398-401, 2010.

46. Lieber RL, Yeh Y, and Baskin RJ. Sarcomere length determination using laser diffraction. Effect of beam and fiber diameter. Biophys J 45: 1007-1016, 1984.

47. Lynn $\mathbf{R}$ and Morgan DL. Decline running produces more sarcomeres in rat vastus intermedius muscle fibers than does incline running. J Appl Physiol 77: 1439-1444, 1994.

48. Lynn R, Talbot JA, and Morgan DL. Differences in rat skeletal muscles after incline and decline running. J Appl Physiol 85: 98-104, 1998.

49. Maréchal G and Plaghki L. The deficit of the isometric tetanic tension redeveloped after release of frog muscle at a constant velocity. J Gen Physiol 73: 453-467, 1979.

50. Meijer K. History dependence of force production in submaximal stimulated rat medial gastrocnemius muscle. J Electromyogr Kinesiol 12: 463-470, 2002.

51. Morais GP, da Rocha AL, Neave LM, Lucas GD, Leonard TR, Carvalho A, da Silva AS, Herzog W. Chronic uphill and downhill exercise protocols do not lead to sarcomerogenesis in mouse skeletal muscle. Journal of Biomechanics. 2019 (In Press)

52. Morgan DL, Whitehead NP, Wise AK, Gregory JE, and Proske U. Tension changes in the cat soleus muscle following slow stretch or shortening of the contracting muscle. $J$ Physiol 522: 503-512, 2000.

53. Nicolopoulos-Stournaras $\mathbf{S}$ and Iles JF. Hindlimb muscle activity during locomotion in the rat (Rattus norvegicus) (Rodentia: Muridae). J Zool 203: 427-440, 1984.

54. Nishikawa KC, Monroy JA, Uyeno TE, Yeo SH, Pai DK, and Lindstedt SL. Is titin a 'winding filament'? A new twist on muscle contraction. Proc R Soc B 279: 981-990, 2012.

55. Noble MIM. Enhancement of mechanical performance of striated muscle by stretch during contraction. Exp Biol 77: 539-552, 1992.

56. Paquin $\mathbf{J}$ and Power GA. The history dependence of the EMG-torque relationship. $J$ Electromyogr Kinesiol 41: 109-115, 2018.

57. Pinnell RAM, Mashouri PM, Mazara N, Weersink E, Brown SHM, and Power GA. Residual force enhancement and force depression in human single fibres. J Biomech 91: 164-169, 2019. 
58. Power GA, Herzog W, and Rice CL. Decay of force transients following active stretch is slower in older than young men: Support for a structural mechanism contributing to force enhancement in old age. J Biomech 47: 3423-3427, 2014a.

59. Power GA, Makrakos DP, Rice CL, and Vandervoort AA. Enhanced force production in old age is not a far stretch: an investigation of residual force enhancement and muscle architecture. Physiol Rep 1: e00004, 2013.

60. Power GA, Makrakos DP, Stevens DE, Herzog W, Rice CL, and Vandervoort AA. Shortening induced torque depression in old men: implications for age-related power loss. Exp Gerontol 57: 75-80, 2014b.

61. Power GA, Rice CL, and Vandervoort AA. Increased residual force enhancement in older adults is associated with a maintenance of eccentric strength. PLoS One 7: e48044, 2012a.

706

707

708

62. Power GA, Rice CL, and Vandervoot AA. Residual force enhancement following eccentric induced muscle damage. J Biomech 45: 1835-1841, $2012 \mathrm{~b}$.

63. Prado L, Makarenko I, Andresen C, Kruger M, Opitz CA, and Linke WA. Isoform diversity of giant proteins in relation to passive and active contractile properties of rabbit skeletal muscle. J Gen Phys 126:461-480, 2005.

64. Pun C, Syed A, and Rassier DE. History-dependent properties of skeletal muscle myofibrils contracting along the ascending limb of the force-length relationship. Proc Biol Sci 277: 475-484, 2010.

65. Ramsey KA, Bakker AJ, and Pinniger GJ. Fiber-type dependence of stretch-induced force enhancement in rat skeletal muscle. Muscle Nerve 42: 769-777, 2010.

66. Ranatunga $\mathbf{K}$ and Thomas $\mathbf{P}$. Correlation between shortening velocity, force-velocity relation and histochemical fibre-type composition in rat muscles. J Muscle Res Cell Motil 11: 240-250, 1990.

67. Rassier DE and Herzog W. Active force inhibition and stretch-induced force enhancement in frog muscle treated with BDM. J Appl Physiol 97: 1395-1400, 2004a.

68. Rassier DE and Herzog W. Considerations on the history dependence of muscle contraction. J Appl Physiol 96: 419-427, 2004b.

69. Rassier DE and Herzog W. Relationship between force and stiffness in muscle fibers after stretch. J Appl Physiol 99: 1769-1775, 2005.

70. Rassier DE, Herzog W, and Pollack GH. Dynamics of individual sarcomeres during and after stretch in activated single myofibrils. Proc Biol Sci 22: 1735-1740, 2003.

71. Reeves ND, Maganaris CN, Longo S, and Narici MV. Differential adaptations to eccentric versus conventional resistance training in older humans. Exp Physiol 94: 825833, 2009.

72. Rode C, Siebert T, and Blickhan R. Titin-induced force enhancement and force depression: a 'sticky-spring' mechanism in muscle contractions? J Theor Biol 259: 350360, 2009.

73. Roots H, Pinniger CJ, Offer GW, and Ranatunga KW. Mechanisms of force enhancement during and after lengthening of active muscle: a temperature dependence study. J Muscle Res Cell Motil 33: 313-325, 2012.

74. Seiberl W, Power GA, and Hahn D. Residual force enhancement in humans: Current evidence and unresolved issues. J Electromyogr Kinesiol 25: 571-580, 2015a. 
75. Sharifnezhard A, Marzilger R, and Arampatzis A. Effects of load magnitude, muscle length and velocity during eccentric chronic loading on the longitudinal growth of the vastus lateralis muscle. J Exp Biol 217: 2726-2733, 2014.

76. Siebert T, Kurch D, Blickhan R, and Stutzig N. Does weightlifting increase residual force enhancement? J Biomech 49: 2047-2052, 2016.

77. Stephenson DG and Williams DA. Effects of sarcomere length on the force-pCa relation in fast- and slow-twitch skinned muscle fibres from the rat. J Physiol 333: 637-653, 1982.

78. Sugi $\mathbf{H}$ and Tsuchiya T. Stiffness changes during enhancement and deficit of isometric force by slow length changes in frog skeletal muscle fibres. J Physiol 407: 215-229, 1988.

79. Timmins RG, Shield AJ, Williams MD, Lorenzen C, and Opar DA. Architectural adaptations of muscle to training and injury: a narrative review outlining the contributions by fascicle length, pennation angle and muscle thickness. Br J Sports Med 50: 1467-1472, 2016.

80. Wigston D and English A. Fibre-type proportions in mammalian soleus muscle during postnatal development. J Neurobiol 23: 61-70, 1991. 


\section{Trainability of the history dependence of force}

755 Table 1. Force and work values across the various training groups and protocols for the SOL and 756 EDL ( $n=31$ male rats). Isometric force was not significantly different across muscles $(p=0.203)$ 757 or training groups $(p=0.905)$. Peak eccentric force was $61 \%$ greater for the SOL than EDL. Work 758 of shortening was 37\% greater for the SOL than EDL $(p<0.001)$ and $15 \%$ greater for the fixed 759 compared to relative protocol $(p=0.044)$. Normalized values of isometric force, eccentric force, 760 and work of shortening were 34\%, 96\%, and 65\% greater for the SOL than EDL, respectively ( $p$ $761<0.05)$.

762

763

\begin{tabular}{|c|c|c|c|c|c|c|}
\hline & \multicolumn{3}{|c|}{ Soleus (SOL) } & \multicolumn{3}{|c|}{ Extensor Digitorum Longus (EDL) } \\
\hline & Control & Uphill & Downhill & Control & Uphill & Downhill \\
\hline Iso $\mathrm{f}$ & $1.39 \pm 0.07$ & $1.21 \pm 0.15$ & $1.23 \pm 0.17$ & $1.10 \pm 0.08$ & $1.21 \pm 0.08$ & $1.15 \pm 0.13$ \\
\hline Iso force, $\mathrm{N} / \mathrm{cm}^{2 *}$ & $9.51 \pm 0.87$ & $7.95 \pm 1.47$ & $9.03 \pm 1.31$ & $6.41 \pm 0.66$ & $6.95 \pm 0.87$ & $6.43 \pm 0.89$ \\
\hline Fixed Protocol & Control & Uphill & Downhill & Control & Uphill & Downhill \\
\hline Ecc force, $\mathrm{N}^{*}$ & $2.44 \pm 0.12$ & $2.25 \pm 0.25$ & $2.29 \pm 0.19$ & $1.22 \pm 0.14$ & $1.47 \pm 0.12$ & 0.20 \\
\hline Ecc force, $\mathrm{N} / \mathrm{cm}^{2 *}$ & $16.78 \pm 1.55$ & $14.38 \pm 2.23$ & $16.90 \pm 1.73$ & $7.01 \pm 0.91$ & $8.32 \pm 0.99$ & 1.31 \\
\hline Con work, mJ*† & $3.34 \pm 0.22$ & $2.99 \pm 0.41$ & $3.00 \pm 0.43$ & $1.96 \pm 0.18$ & $2.19 \pm 0.19$ & $2.23 \pm 0.28$ \\
\hline Con work, $\mathrm{mJ} / \mathrm{cm}^{2 *}$ & $23.10 \pm 2.46$ & $19.82 \pm 3.89$ & $22.06 \pm 3.48$ & $11.37 \pm 1.31$ & $12.68 \pm 1.90$ & $12.59 \pm 1.88$ \\
\hline Relative Protocol & Control & Uphill & Downhill & Control & Uphill & Downhill \\
\hline Ecc force, $\mathrm{N}^{*}$ & $2.23 \pm 0.14$ & $2.12 \pm 0.29$ & $2.07 \pm 0.21$ & $1.26 \pm 0.16$ & $1.39 \pm 0.12$ & 0.22 \\
\hline Ecc force, $N / \mathrm{cm}^{2 *}$ & $15.48 \pm 1.71$ & $13.82 \pm 2.62$ & $15.37 \pm 1.91$ & $7.32 \pm 1.12$ & $7.86 \pm 1.04$ & $8.54 \pm 1.46$ \\
\hline Con work, mJ*† & $2.76 \pm 0.19$ & $2.35 \pm 0.39$ & $2.47 \pm 3.57$ & $1.85 \pm 0.17$ & $2.12 \pm 0.27$ & $2.09 \pm 0.32$ \\
\hline Con work, $\mathrm{mJ} / \mathrm{cm}^{2 *}$ & $19.07 \pm 2.00$ & $15.69 \pm 3.63$ & $18.19 \pm 2.88$ & $10.77 \pm 1.29$ & $12.34 \pm 2.20$ & $11.92 \pm 2.04$ \\
\hline
\end{tabular}

764 Values are means \pm SE. * Indicates an effect of muscle $(p<0.05)$. $\dagger$ Indicates an effect of protocol 765 ( $p$ 0.05). Iso force, isometric force at optimal muscle length; Ecc force, peak eccentric force; 766 Con work, work of shortening. 
769 Table 2. Muscle length measures across the various training groups for the SOL and EDL $(n=30$ 770 male rats). The SOL had a $17 \%$ shorter muscle $(p<0.001)$ but $13 \%$ longer fascicles $(p<0.001)$ 771 compared to the EDL. However, there was no effect of training for either muscle length or fascicle 772 length $(p \geq 0.05)$.

773

\begin{tabular}{ccccccc}
\hline \hline & \multicolumn{3}{c}{ Soleus (SOL) } & \multicolumn{3}{c}{ Extensor Digitorum Longus (EDL) } \\
\cline { 2 - 7 } & Control & Uphill & Downhill & Control & Uphill & Downhill \\
\hline SL, $\mu \mathrm{m}$ & $2.75 \pm 0.11$ & $2.76 \pm 0.14$ & $2.77 \pm 0.09$ & $2.75 \pm 0.05$ & $2.62 \pm 0.11$ & $2.69 \pm 0.11$ \\
FL, mm* & $18.60 \pm 0.32$ & $18.53 \pm 0.51$ & $19.33 \pm 0.26$ & $16.27 \pm 0.51$ & $16.89 \pm 0.54$ & $16.94 \pm 0.26$ \\
$\mathrm{~L}_{0}, \mathrm{~mm}^{*}$ & $30.75 \pm 0.86$ & $30.62 \pm 0.73$ & $31.08 \pm 0.51$ & $36.88 \pm 0.67$ & $37.27 \pm 0.86$ & $37.70 \pm 0.63$ \\
\hline
\end{tabular}

774 Values are means \pm SE. * Indicates an effect of muscle $(p<0.05)$. SL, average sarcomere length;

775 FL, fascicle length; Lo, optimal muscle length. 
Trainability of the history dependence of force

\section{Figure Captions}

Figure 1: Schematic experimental timeline. A. Determination of twitch current. B. Determination of $\mathrm{L}_{0} / \mathrm{F}_{0}$. C. Fixed rFD protocol. D. Relative rFD protocol. E. Fixed rFE protocol. F. Relative rFE protocol. G. Final muscle assessment. The fixed and relative protocols were randomized. $L_{o}$, optimal muscle length; $F_{o}$, isometric force at optimal muscle length; $r F D$, residual force depression; ISO.S, short reference isometric; ISO.L, long reference isometric; rFE, residual force enhancement.

Figure 2: Sarcomerogenesis following uphill and downhill running. Mean $\pm 95 \%$ confidence intervals for SSN ( $n=31$ male rats). There was an interaction of muscle $\times$ training $(p<0.001)$ for SSN, in addition to main effects of muscle $(p<0.001)$ and training $(p=0.004)$. SSN within SOL were $6 \%$ greater for the downhill compared to uphill group $(p=0.003)$, and within the EDL there was a trend $(p=0.056)$ towards $4 \%$ more SSN for the downhill compared to control. * Indicates a significant difference $(p<0.05)$. SSN, serial sarcomere numbers; SOL, soleus; EDL, extensor digitorum longus.

Figure 3: Residual force depression. A. Experimental data traces from the soleus, with the rFD contraction in gray and ISO.S contraction in black. Note the comparatively lower isometric force in the rFD condition for the same muscle length (and level of activation). B. Absolute rFD values across the various training groups and protocols for the SOL and EDL ( $n=31$ male rats). Absolute rFD was $45 \%$ greater for the SOL than EDL $(p<0.001)$ but was not significantly different across training groups $(p=0.645)$ or protocols $(p=0.098)$. C. Normalized rFD values across the various training groups and protocols for the SOL and EDL $(n=30$ male rats). Normalized rFD was $2.1 \times$ greater $(p<0.001)$ for the EDL than SOL but was not significantly different across training groups $(p=0.745)$ or protocols $(p=0.219)$ ( $n=31$ male rats). D. Relationship between specific work of shortening and absolute rFD values across training groups during the fixed protocol for the SOL (black) and EDL (white) ( $n=31$ male rats). Specific work of shortening was positively and linearly associated with absolute rFD for both the SOL $\left(\mathrm{R}^{2}=0.71, p<0.001\right)$ and EDL $\left(\mathrm{R}^{2}=0.61, p<\right.$ $0.001)$. * Indicates a significant relationship $(p<0.05)$.Values are means \pm SE. * Indicates an effect of muscle $(p<0.05)$. ISO.S, short reference isometric; $r F D$, residual force depression; $S O L$, soleus; EDL, extensor digitorum longus.

Figure 4: Residual force enhancement. A. Experimental data traces from the soleus, with the rFE contraction in gray and ISO.L contraction in black. Note the comparatively higher isometric force in the rFE condition (and comparatively higher passive force in the pFE condition) for the same muscle length (and level of activation). B. Absolute rFE values across the various training groups and protocols for the SOL and EDL ( $n=31$ male rats). Absolute rFE was 2.3× greater for the SOL than EDL $(p<0.001)$ and $56 \%$ greater for the fixed compared to relative protocol $(p=$ $0.004)$, with no significant difference across training groups $(p=0.929)$. C. Normalized rFE values across the various training groups and protocols for the SOL and EDL ( $n=30$ male rats). Normalized rFE was $43 \%$ greater for the fixed compared to relative protocol $(p=0.009)$ but was not significantly different across muscles $(p=0.386)$ or training groups $(p=0.450)$. D. Relationship between specific eccentric force and absolute rFE values across training groups during the fixed protocol for the SOL (black) and EDL (white) ( $n=31$ male rats). Specific eccentric force was positively and linearly associated with absolute rFE for both the SOL $\left(\mathrm{R}^{2}=\right.$ 


\section{Trainability of the history dependence of force}

825 0.57, $p<0.001)$ and EDL $\left(\mathrm{R}^{2}=0.73, p<0.001\right) . r F E$, residual force enhancement; ISO.L, long 826 reference isometric; $p F E$, passive force enhancement. Values are means $\pm \mathrm{SE}$. ${ }^{*}$ Indicates an 827 effect of muscle $(p<0.05)$. $†$ Indicates an effect of protocol $(p<0.05)$.

Figure 5: Passive force enhancement. A. Absolute $\mathrm{pFE}$ values across the various training groups and protocols for the SOL and EDL ( $n=31$ male rats). Absolute $\mathrm{pFE}$ was $2.2 \times$ greater for the SOL than EDL $(p<0.001)$ but was not significantly different across training groups $(p=0.320)$ or protocols $(p=0.225)$. B. Normalized $p F E$ values across the various training groups and protocols for the SOL and EDL ( $n=30$ male rats). Normalized pFE was 3.3× greater for the SOL than EDL $(p<0.001)$ but was not significantly different across training groups $(p=0.320)$ or protocols $(p=$ 0.700). C. Relationship between absolute $\mathrm{pFE}$ and absolute rFE values across training groups during the fixed protocol for the SOL (black) and EDL (white) ( $n=31$ male rats). Absolute pFE was positively and linearly associated with absolute rFE for both the $\mathrm{SOL}\left(\mathrm{R}^{2}=0.41, p<0.001\right)$ and EDL $\left(\mathrm{R}^{2}=0.69, p<0.001\right)$. D. Relationship between specific eccentric force and absolute pFE values across training groups during the fixed protocol for the SOL (black) and EDL (white) 841 pFE for both the SOL $\left(\mathrm{R}^{2}=0.49, p<0.001\right)$ and EDL $\left(\mathrm{R}^{2}=0.65, p<0.001\right)$. Values are means $842 \pm$ SE. * Indicates an effect of muscle $(p<0.05)$. $p F E$, passive force enhancement; SOL, soleus; 843 EDL, extensor digitorum longus. 
Figure 1.

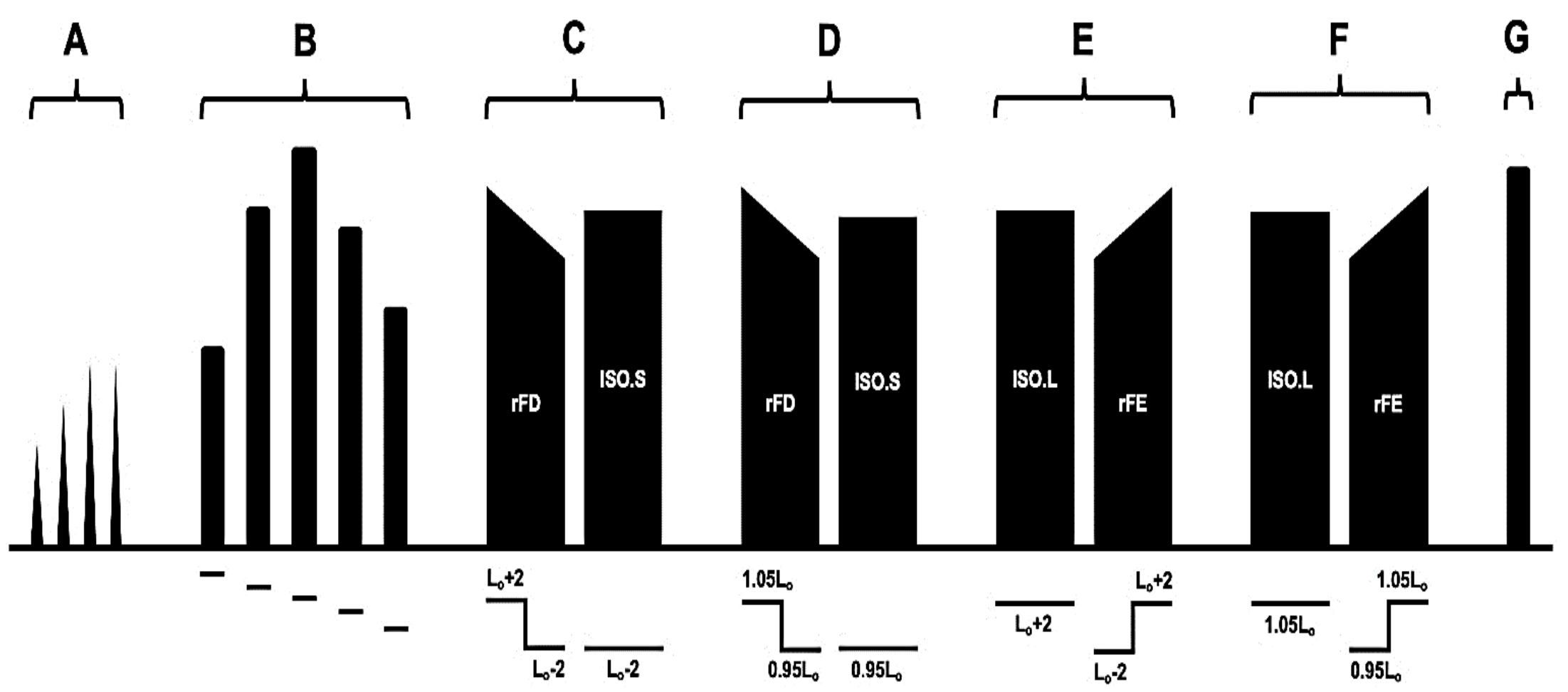


Figure 2.

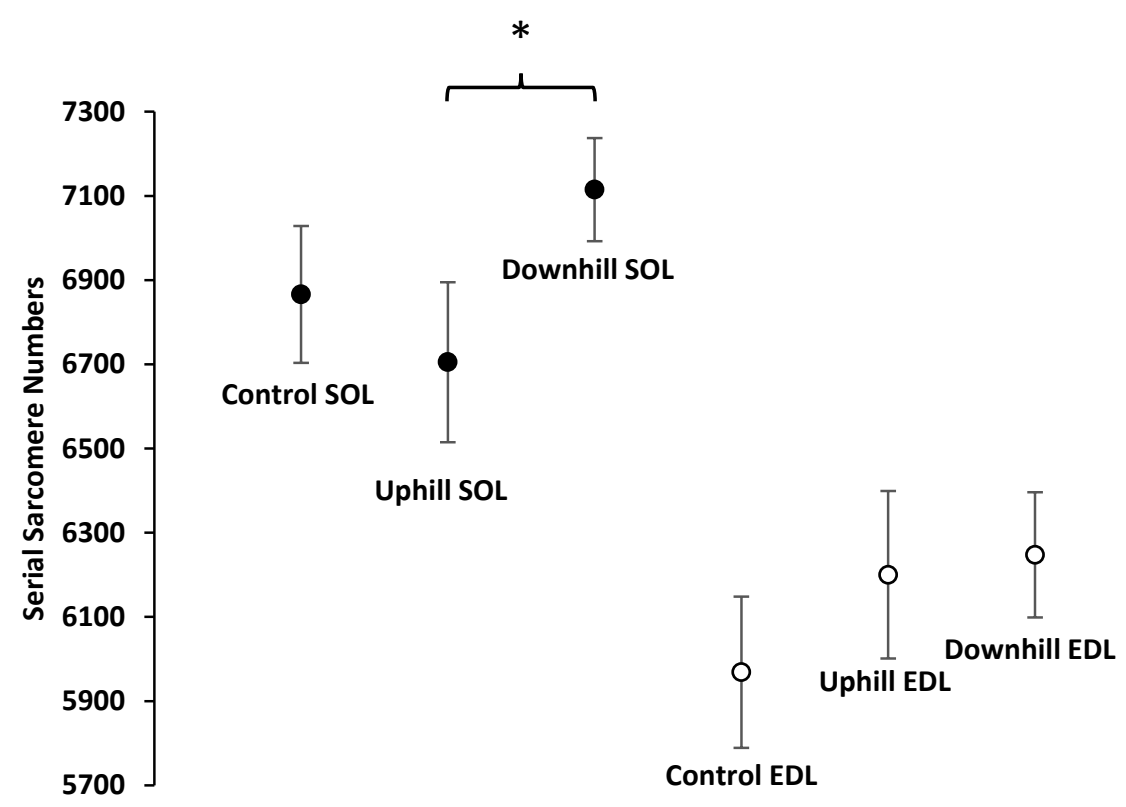


Figure 3.

A.

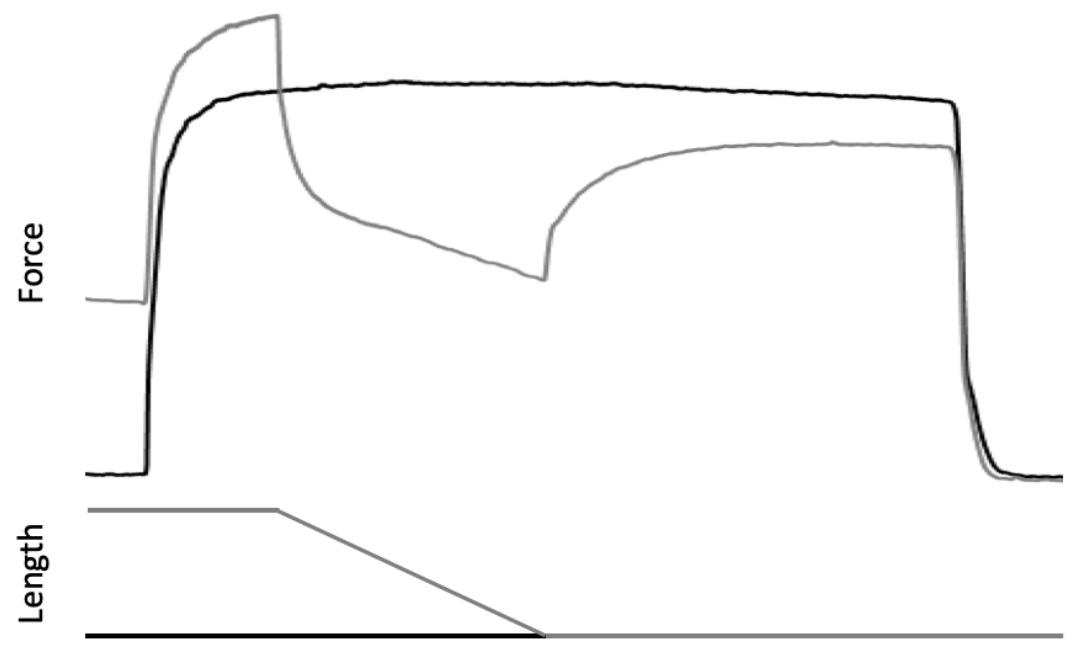

$1 \mathrm{~s}$

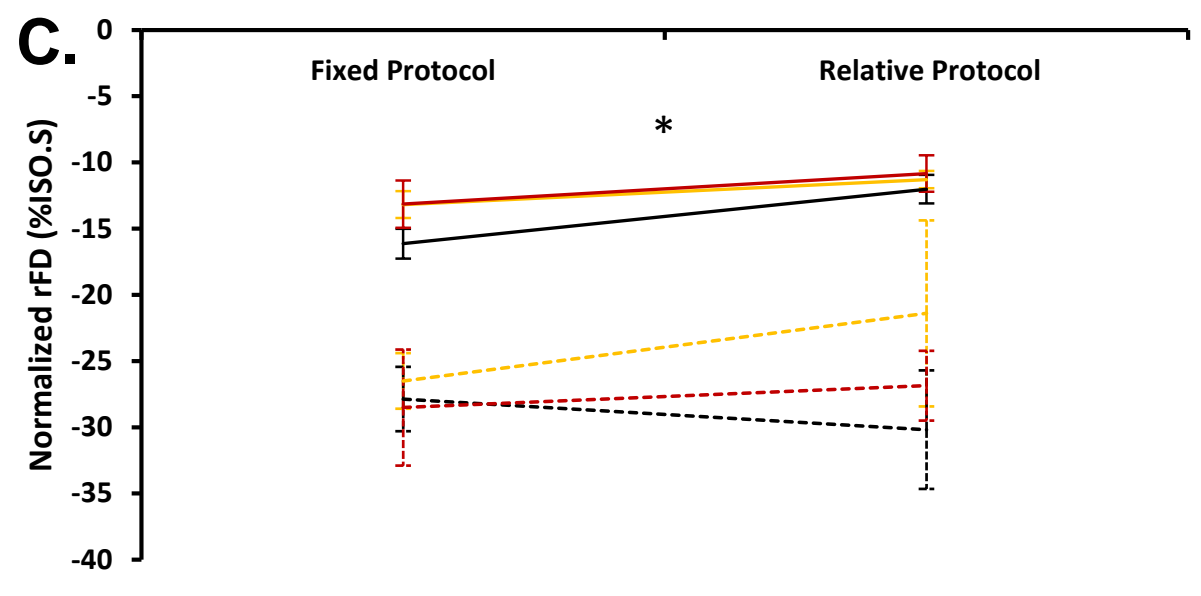

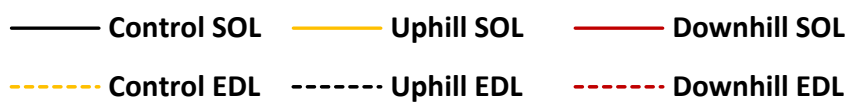

B.
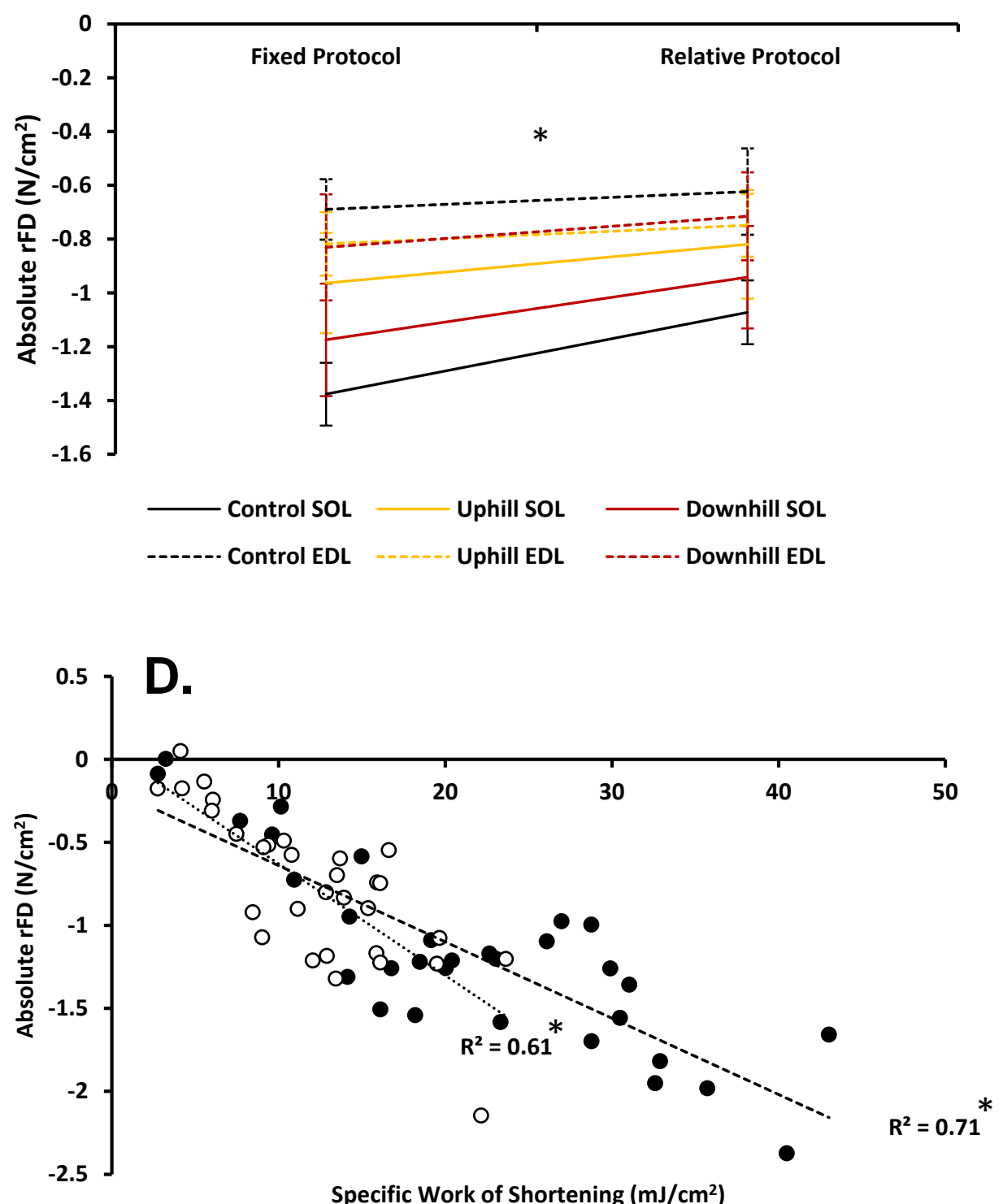


\section{Figure 4.}
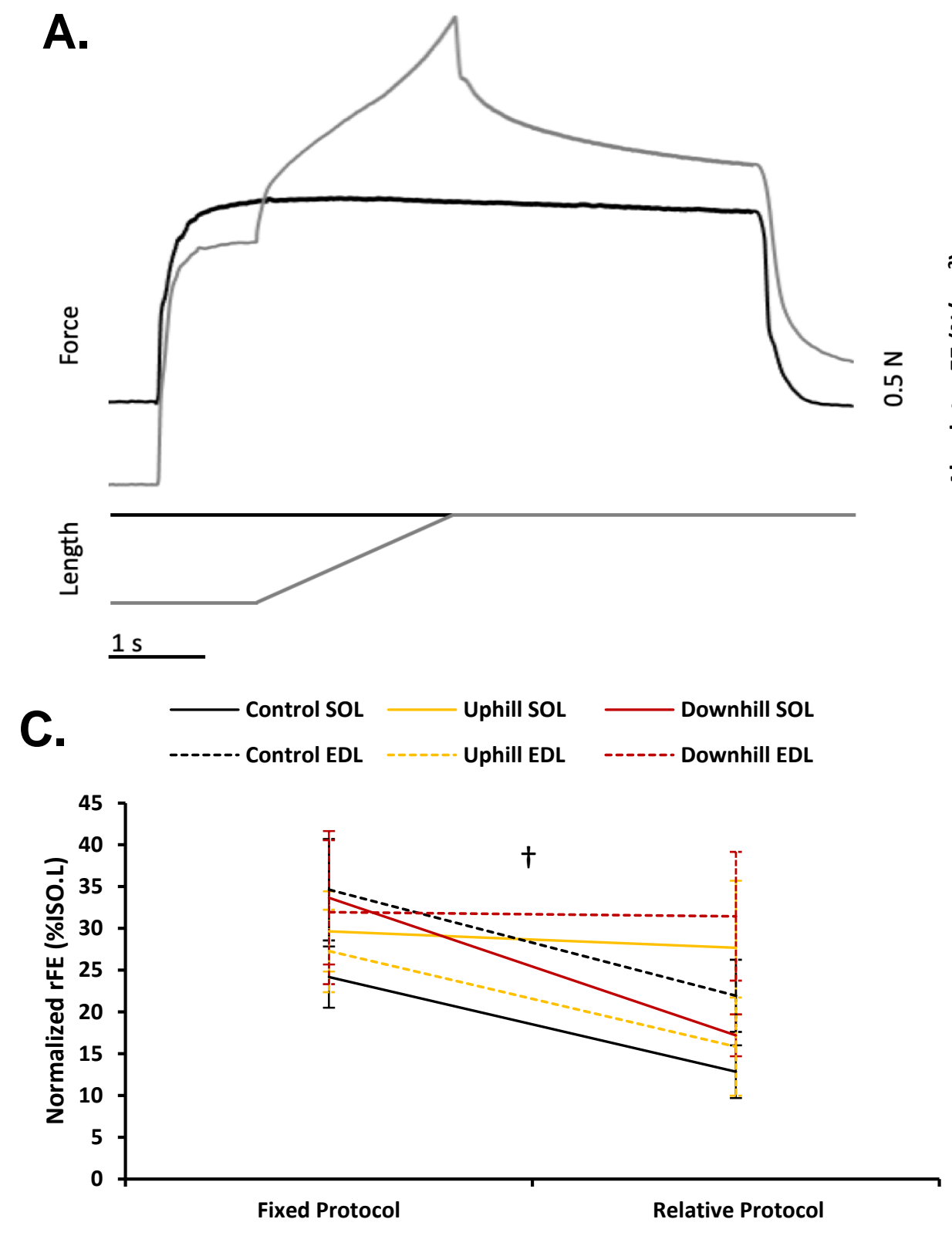

B.

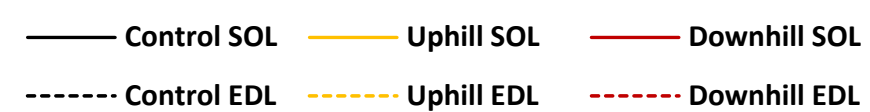

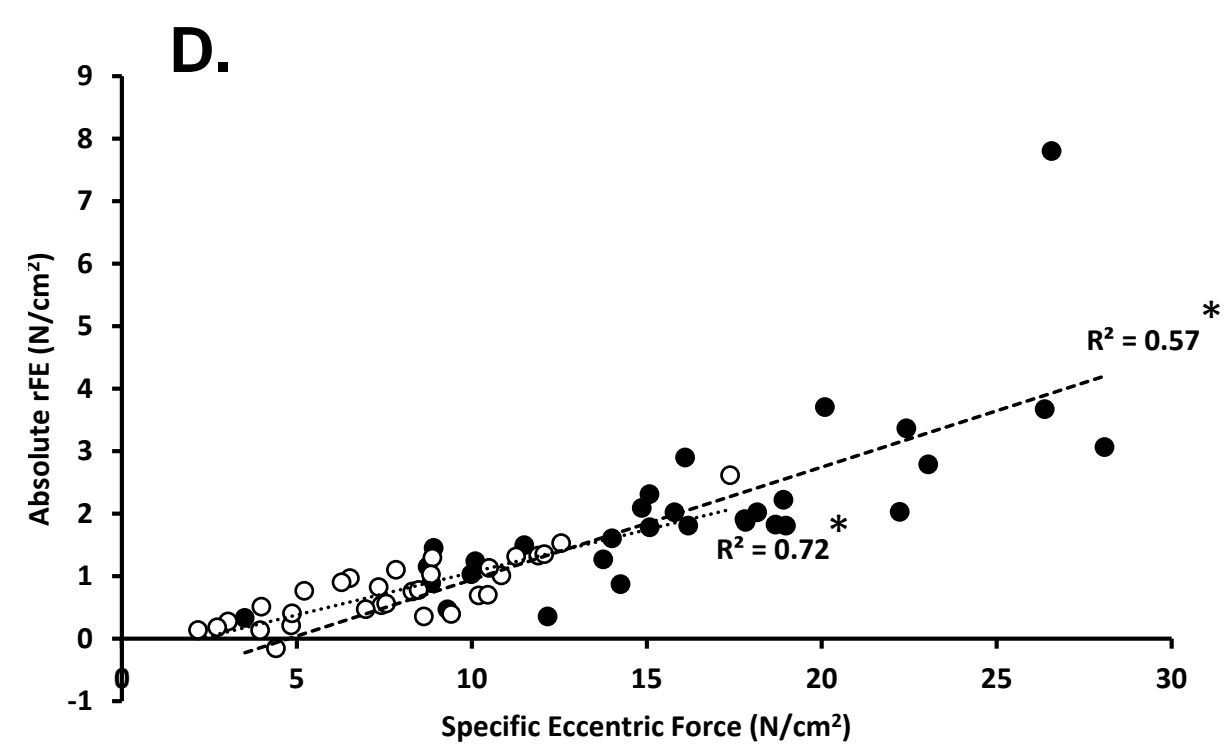




\section{Figure 5.}

A.
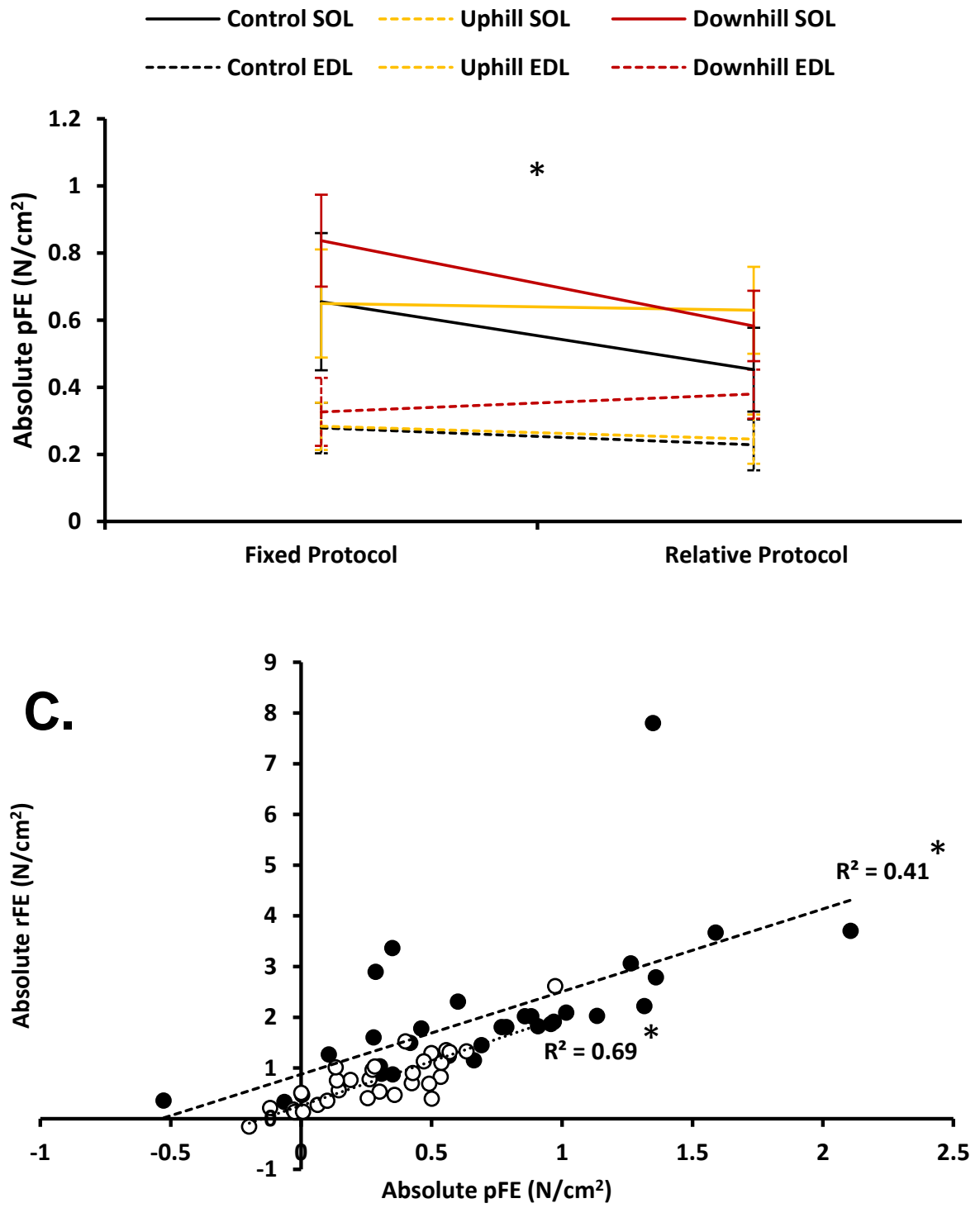

B.

Control SOL Uphill SOL — Downhill SOL

Control EDL _..... Uphill EDL _-.... Downhill EDL
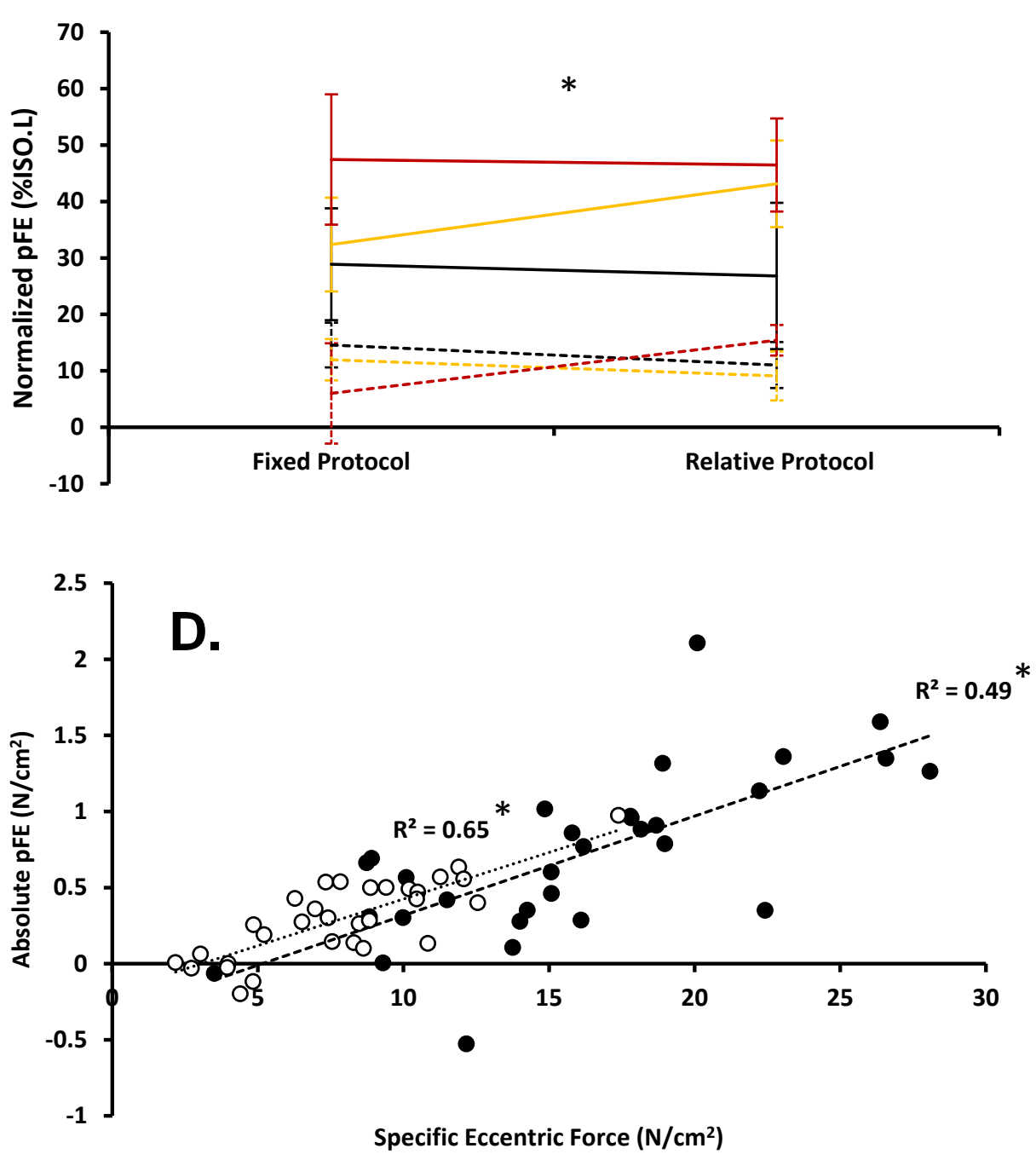